\title{
Esterification reactions catalyzed by lipases immobilized in organogels: effect of temperature and substrate diffusion
}

\author{
M. Zoumpanioti · P. Parmaklis • \\ P. Domínguez de María $\cdot$ H. Stamatis $\cdot$ \\ J. V. Sinisterra $\cdot$ A. Xenakis
}

Received: 18 February 2008/Revised: 24 March 2008/Accepted: 31 March 2008

(C) Springer Science+Business Media B.V. 2008

\begin{abstract}
Rhizomucor miehei lipase was immobilized in hydroxy(propylmethyl) cellulose or agar gels containing lecithin or AOT microemulsions. The effect of the diffusion of substrates and products to this catalyst was studied, as well as the effect of temperature on the initial rate of ester synthesis. The composition of the gel affects the reaction rate due to mass transport phenomena. The apparent activation energies were higher for the systems based on agar, independently of the microemulsion used, and lower for the systems based on AOT microemulsions, independently of the polymer used.
\end{abstract}

Keywords Activation energy - Catalysis · Diffusion · Lipase · Organogels · Temperature

M. Zoumpanioti · P. Parmaklis · A. Xenakis $(\square)$ Institute of Biological Research and Biotechnology, National Hellenic Research Foundation, Athens, Greece e-mail: arisx@eie.gr

P. D. de María · J. V. Sinisterra

Biotransformations Group, Faculty of Pharmacy, Universidad Complutense, Madrid, Spain

H. Stamatis

Biological Applications and Technologies Department, University of Ioannina, Ioannina, Greece

\section{Introduction}

Microemulsions are efficient media for achieving enzymatic esterifications (Fletcher et al. 1985; Rees and Robinson 1993; Oldfield 1994; Stamatis et al. 1999) as they can simultaneously solubilize hydrophilic, hydrophobic or amphiphilic molecules. Enzymes hosted in the aqueous core of water in oil microemulsions can maintain their catalytic activity and even favour achieving synthetic reactions instead of hydrolytic ones (Nascimento et al. 1992; Backlund et al. 1995; Rees et al. 1995). However, microemulsions contain surfactants, which is a major barrier on applying many physicochemical techniques for efficiently separating and isolating the product as well as retrieving the catalyst.

To overcome this problem, microemulsion-based gels are suggested as alternative media for enzyme immobilization. These gels provide the advantage of a "double" immobilization of the enzyme, primary into the microemulsion droplets and secondly on the "network" of the gelling agent. They are stable in several organic polar or non-polar solvents (Delimitsou et al. 2002; Blattner et al. 2006) and are successfully used for esterification of fatty acids as well as phenolic acids with aliphatic alcohols (Delimitsou et al. 2002; Zoumpanioti et al. 2006).

The enzyme-containing organogels are actually a solid catalyst which is used to catalyze reactants dissolved in organic solvents. Substrates present in the organic solution become available for reaction 
when they diffuse to the interior of the gel and come closer to the enzyme microenvironment (Rees et al. 1991). So, in order to establish the organogels as appropriate media for enzymatic catalysis it must be clarified whether the diffusion of substrates and products into the catalyst is feasible or whether the energy that is needed in order to achieve the reaction is a barrier to the gel's use as a solid catalyst. In this respect, studying the effect of temperature on the enzyme kinetics can reveal useful information on the activation energy of the catalytic process.

\section{Materials and methods}

\section{Materials}

Lipase from Rhizomucor miehei was supplied by Fluka. Its specific activity was $2.3 \mathrm{U} / \mathrm{mg}$ of protein ( $1 \mathrm{U}$ corresponds to the amount of enzyme which liberates $1 \mu \mathrm{mol}$ butyric acid per min at $\mathrm{pH} 8.0$ and $40^{\circ} \mathrm{C}$ using tributyrine as substrate). AOT, lecithin, containing $40 \% \quad(\mathrm{w} / \mathrm{w})$ phosphatidylcholine, and hydroxy(propylmethyl) cellulose (HPMC) were purchased from Sigma. All other reagents were of the highest commercially available purity.

Preparation of microemulsions and organogels

AOT and lecithin microemulsions were prepared as described elsewhere (Delimitsou et al. 2002). Lipasecontaining microemulsion-based organogels (MBGs) were prepared by adding appropriate amounts of lipase-containing microemulsion $(0.2 \mathrm{mg}$ lipase $/ \mathrm{ml}$ of microemulsion) to a second solution of polymer in water (Delimitsou et al. 2002).

\section{Diffusion of substrates and products}

HPMC and agar organogels based on lecithin or AOT microemulsions were prepared in absence of enzyme and placed in sealed vials with $10 \mathrm{ml}$ isooctane that already contained the reactant or ester in varied concentrations. At fixed time intervals $10 \mu 1$ samples were withdrawn and directly analysed by gas chromatography.
Effect of incubation temperature on reaction kinetics

The determination of lipase activity was based on the measurement of the initial rate of lauric acid ester synthesis. Four millilitre HPMC MBG or $6.5 \mathrm{ml}$ agar MBG were placed into a reaction vial. The reaction was initiated by adding $10 \mathrm{ml}$ isooctane containing 1propanol $(100 \mathrm{mM})$ and lauric acid $(100 \mathrm{mM})$. The reaction was held at the stated temperature and the conversion was monitored by GC (Stamatis and Xenakis 1999).

Determination of activation energy

The activation energy $\left(E_{a}\right)$ was determined using the Arrhenius equation:

$k=A e^{-E_{a} / R T}$

where $k$ is the rate constant of the reaction, $A$ the Arrhenius pre-exponential factor, $R$ the gas constant (1.987 $\mathrm{cal} \mathrm{K}^{-1} \mathrm{~mol}^{-1}$ ) and $T$ the absolute temperature $(\mathrm{K})$.

\section{Results and discussion}

Diffusion of substrates and products

As the biocatalytic procedure involving MBGs is heterogeneous, it would be necessary to study the diffusion of the substrates and products to the biocatalyst that can limit the esterification rate. The diffusion of the substrates and products in the absence of enzyme, more specifically lauric acid, 1-heptanol and butyl laurate, were evaluated in agar and HPMC organogels based on AOT or lecithin microemulsions.

Figure 1 shows that the concentration of 1-heptanol (in the absence of acid) and lauric acid (in the absence of alcohol) in the external solvent decreases until equilibrium is established between the alcohol/ acid in the gel and the alcohol/acid in the solvent. Equilibrium is reached for every initial concentration studied.

After equilibrium is settled, the concentration of the alcohol in the interior of the MBGs seems to be constant and equal to $\approx 4 \mathrm{mM}$ in all cases of AOT 


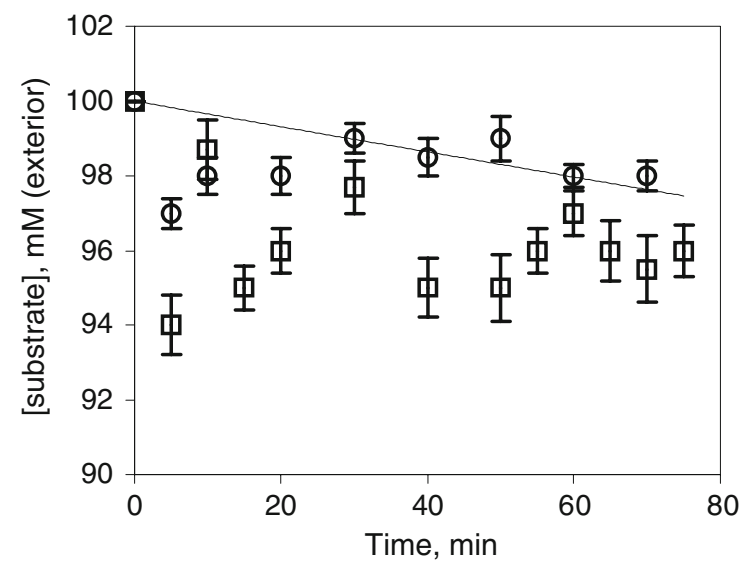

Fig. 1 Diffusion at $25^{\circ} \mathrm{C}$ of 1-heptanol towards the interior of HPMC organogels based on lecithin microemulsion $(\bigcirc)$ and lauric acid towards the interior of HPMC organogels based on AOT microemulsion ( $\square$ ). Initial substrates concentration in the external solvent (isooctane) $100 \mathrm{mM}$; solvent volume $10 \mathrm{ml}$

MBGs, independently on the initial alcohol concentration. Although the percentage of alcohol that remains in the external solvent is different in each case (different initial alcohol concentrations), the amount of alcohol in the interior of the gel seams to be always the same $(4 \mathrm{mM})$. In the case of lecithin MBGs the alcohol concentration in the interior of the gel after the equilibrium is settled is also constant but lower $(1.5-2 \mathrm{mM})$. This indicates that the surfactant used for the formation of the MBG influences the structure of the organogel produced.

The acid concentration in the interior of the gel after equilibrium is settled is also constant and close to $4 \mathrm{mM}$. Moreover, the study of the diffusion of butyl laurate in HPMC gels based on various microemulsions (data not shown) indicates that when the gels get in contact with the organic solvent, the concentration of the ester in the organic solvent decreases by $20 \%$ and further increases so that the equilibrium is quickly settled. In all MBGs studied, the amount of ester that remains in the external organic solvent after the equilibrium is $97-99 \%$ of its initial concentration.

The structure of the MBG permits the diffusion of the substrates as well as the products, since the ester-after equilibrium is settled-remains in a high ratio outside the MBG. Therefore, the structure of the MBGs does not provide a barrier for the reactants to enter the catalyst, nor keep the product inside; hence, they can be considered as appropriate media for esterification reactions of fatty acids with aliphatic alcohols.

During a biocatalytic synthesis we can assume that the alcohol concentration achieved in the interior of the gel remains constant, except for the case of a too high reaction rate where the slower diffusion cannot set the equilibrium concentration so quickly. The concentration of the substrates into the gel tends to zero since as soon as they reach the enzyme they are instantly converted. In this case the kinetic procedure is therefore diffusion limited. On the other hand, if the synthesis takes place with a lower rate, then there is enough time for the equilibrium concentration in the interior of the catalyst to settle, and the synthesis is limited by the reaction rate. This study is fundamental for all the conversion reactions that involve diffusion of substrates or products at least in one stage of the procedure.

Effect of incubation temperature on reaction kinetics

The activity of $R$. miehei lipase immobilized in agar and HPMC MBGs towards the esterification reaction of lauric acid with 1-propanol was monitored as a function of incubation temperature. The reaction was initiated by addition of the lipase-containing MBG in the reaction solution which had been pre-equilibrated to the desired temperature.

For HPMC-lecithin MBGs, increasing temperature from 20 to $65^{\circ} \mathrm{C}$, results in increase of reaction's initial rate from 0.9 to $1.8 \mathrm{mM} / \mathrm{min}$ (Fig. 2). However, in the case of HPMC-AOT MBGs, the increase of temperature from 20 to $60^{\circ} \mathrm{C}$ increases the reaction rate only from 0.2 to $0.3 \mathrm{mM} / \mathrm{min}$. In both cases, no loss of lipase activity was observed within this range of temperatures. Similarly, when lipase is immobilized in agar MBGs with a lecithin microemulsion, the increase of temperature leads to an important increase of the reaction rate whereas, for agar MBGs with an AOT microemulsion the increase is significantly lower. However, for agar organogels a loss of lipase activity can be observed at higher temperatures, which is not the case for the analogue HPMC organogels.

For lecithin MBGs (Fig. 2), an increase of the incubation temperature leads to important increase of the reaction's initial rate for both, HPMC and agar. 


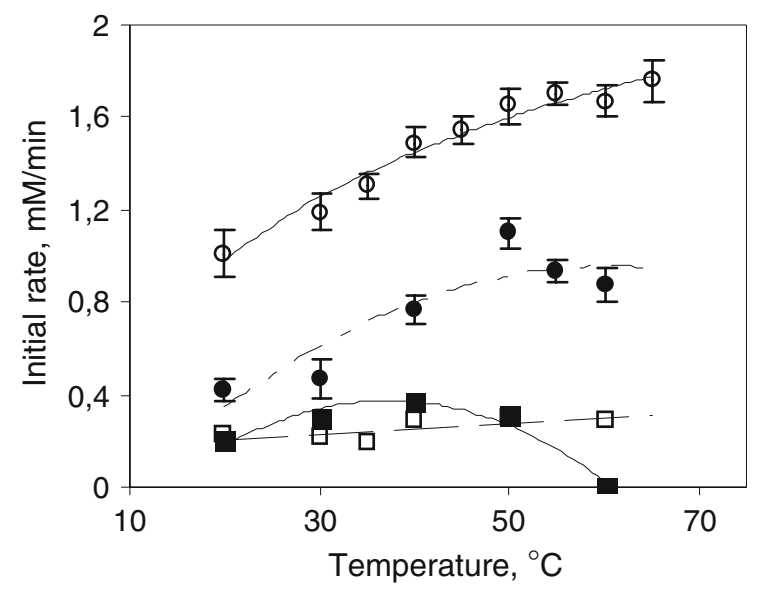

Fig. 2 Effect of temperature on the initial rate of the esterification of lauric acid with 1-propanol catalyzed by $R$. miehei lipase immobilized in HPMC organogels (white symbols) or agar organogels (dark symbols) based on lecithin $(\bigcirc, \boldsymbol{)})$ or AOT $(\square, \mathbf{\square})$ microemulsions. 1-propanol $=100 \mathrm{mM}$; lauric acid $=100 \mathrm{mM}$; isooctane as solvent $(10 \mathrm{ml}) ; R$. miehe $i$ concentration $0.06 \mathrm{mg} / \mathrm{g}$ of gel

The fact that the reaction rate depends strongly on the temperature, suggests that the conversion rate is controlled by the reaction steps, rather than the mass transport phenomena of the diffusion of the substrates in the interior of the catalyst. On the other hand, when the MBGs are based on AOT, only a lower increase of the reaction rate can be monitored upon increase of temperature. Consequently, in AOT MBGs the reaction rate is limited by mass transport phenomena and not by the reaction steps. Therefore, the microemulsion used for gel preparation affects the structure of the gel and influences the reaction rate, with AOT limiting the diffusion of the substrates to the catalyst more strongly than lecithin.

When the lipase is immobilized in HPMC gels it retains its activity through all the temperatures studied (Fig. 2), independently of the microemulsion used. However, in the case of agar MBGs the enzyme loses its activity above $50^{\circ} \mathrm{C}$, either partly $(20 \%$ loss in respect to the maximum activity observed for lecithin-agar gels) or completely $(100 \%$ loss for AOT-agar gels). It should be mentioned here that all the gels studied were stable even at $65^{\circ} \mathrm{C}$, without noticing any change in their structure. Therefore, the loss of activity is probably due to enzyme deactivation and not gel destruction. So, when the lipase is immobilized in HPMC gels it is better protected.
Activation energy

Enzymes are biocatalysts that lower the activation energy $\left(E_{a}\right)$ that is required to convert the substrates to products. In aqueous solutions $E_{a}$ is an intrinsic feature of the enzyme itself (Jenta et al. 1997). However, immobilizing the enzyme, diffusion may become rate limiting and the reaction may switch from kinetic to diffusion controlled resulting in a change in the measured apparent $E_{a}$. Therefore, we determined the activation energy for lipase catalyzed propyl laurate synthesis in HPMC and agar MBGs. The Arrhenius plot for synthesis in HPMC-lecithin gel using $R$. miehei lipase is shown in Fig. 3. The apparent activation energy was calculated for all the gel systems described above (Table 1). The calculated apparent activation energy values vary from 8 to 35 , depending on the composition of the system used.

For the agar gels the apparent activation energy calculated appeared to be higher than the activation

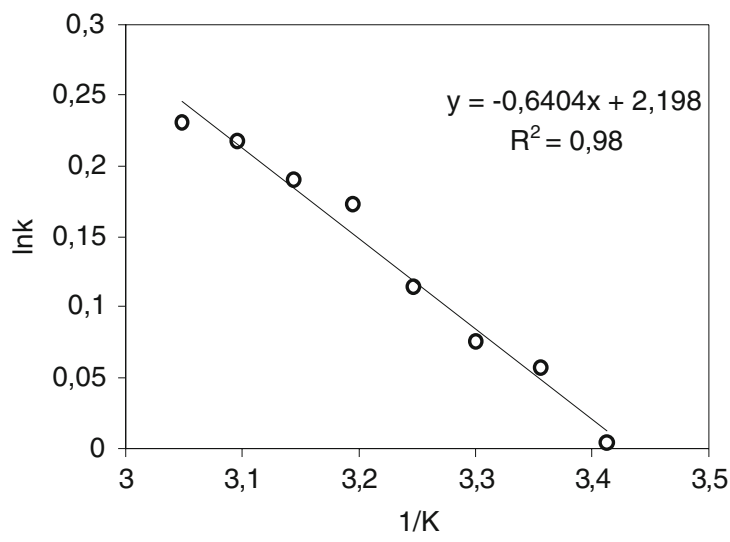

Fig. 3 Arrhenius plot for the esterification reaction in HPMC organogel based on lecithin microemulsion catalyzed by $R$. miehei lipase. The calculated apparent activation energy is $12 \mathrm{~kJ} \mathrm{~mol}^{-1}$. Reaction conditions as given in Fig. 2

Table 1 Apparent activation energies for the enzymatic synthesis of propyl laurate catalyzed by $R$. miehei lipase

\begin{tabular}{llc}
\hline Polymer & Microemulsion & $E_{a}\left(\mathrm{~kJ} \mathrm{~mol}^{-1}\right)^{\mathrm{a}}$ \\
\hline HPMC & Lecithin & 12 \\
HPMC & AOT & 8 \\
Agar & Lecithin & 35 \\
Agar & AOT & 24 \\
\hline
\end{tabular}

${ }^{a}$ Reaction conditions: 1-propanol $=100 \mathrm{mM}$; lauric acid $=$ $100 \mathrm{mM}$; isooctane as solvent $(10 \mathrm{ml}) ; R$. miehei concentration $0.06 \mathrm{mg} / \mathrm{g}$ gel 
energy calculated for the HPMC organogels (Table 1). This implies that in HPMC gels the esterification reaction studied has a lower energy threshold, than in the case of agar organogels. The type of microemulsion used for gel preparation can affect the activation energy of the synthetic reaction, as for both, agar and HPMC MBGs, when lecithin microemulsion is used the activation energy appears to be higher as compared to the one for respective AOT MBGs. This is in agreement with the results of the diffusion study and the temperature effect study presented above.

Finally, it should be noticed that when Candida antarctica lipase was immobilized in the same systems (data not shown), the esterification's activation energy was identical when the enzyme immobilization medium was a HPMC-AOT MBG, as in the case of $R$. miehei lipase. This indicates that mass transport influences the rate of reaction in this system. This is in good agreement with the results of the study for temperature influence on reaction rate presented above. The order of magnitude of the activation energies calculated for all systems studied in this work was similar to that measured by Hedström et al. (2001) for C. antarctica lipase immobilized in organogels. Moreover, the activation energies for MBGs were lower than those calculated for enzymatic reactions in microemulsions in accordance to Biasutti et al. (2008).

\section{Conclusions}

We can assume that both HPMC and agar organogels can be considered as appropriate media for esterification reactions of fatty acids with aliphatic alcohols since the diffusion study has revealed that the structure of the organogels does not provide a barrier for the substrates to enter the catalyst, or keeping the product in it. When the microemulsion used for gel preparation contains lecithin, the esterification rate is kinetically controlled. On the other hand, when the microemulsion used contains AOT the esterification rate is limited by the laws of diffusion, independently of the gelling agent. HPMC organogels provide a lower energy threshold for the esterification reaction compared to agar organogels, for both lecithin and AOT microemulsions. Respectively, AOT microemulsion leads to lower energy threshold compared to lecithin microemulsion, for both HPMC and agar organogels. The order of magnitude of the activation energy calculated for all systems permits their use as media for achieving enzymatic catalysis.

Acknowledgement The Greek General Secretariat for Research and Technology is thanked for the financial support (ПАВЕТ 2006).

\section{References}

Backlund S, Eriksson F, Kanerva L, Rantala M (1995) Selective enzymatic reactions using microemulsion-based gels. Colloids Surf B Biointerfaces 4:121-127

Biasutti MA, Abuin EB, Silber JJ et al (2008) Kinetics of reactions catalyzed by enzymes in solutions of surfactants. Adv Colloid Interface Sci 136:1-24

Blattner C, Zoumpanioti M, Kröner J et al (2006) Biocatalysis using lipase encapsulated in microemulsion-based organogels in supercritical carbon dioxide. J Supercritical Fluids 36:182-193

Delimitsou C, Zoumpanioti M, Xenakis A, Stamatis H (2002) Activity and stability studies of Mucor miehei lipase immobilized in novel microemulsion-based organogels. Biocatal Biotransform 20(5):319-327

Fletcher PDI, Freedman RB, Oldfield C, Robinson BH (1985) Activity of lipase in water-in-oil microemulsions. J Chem Soc Faraday Trans I 81:2667-2679

Hedström G, Backlund S, Eriksson F (2001) Influence of diffusion on the kinetics of an enzyme-catalyzed reaction in gelatine-based gels. J Coll Interface Sci 239:190-195

Jenta TRJ, Batts G, Rees GD, Robinson BH (1997) Kinetic studies of Chromobacterium viscosum lipase in AOT water in oil microemulsions and gelatin microemulsionbased organogels. Biotechnol Bioeng 54:416-427

Nascimento MG, Rezende MC, Vecchia RD et al (1992) Enzyme-catalyzed esterifications in microemulsion-based organo gels. Tetrahedron Lett 33:5891-5894

Oldfield C (1994) Enzymes in water-in-oil microemulsions ('reverced micelles'): principles and applications. Biotechnol Genet Eng Rev 12:255-327

Rees GD, Nascimento MG, Jenta T, Robinson BH (1991) Reverse enzyme synthesis in microemulsion-based organo-gels. Biochim Biophys Acta 1073:493-501

Rees GD, Robinson BH (1993) Microemulsions and organogels: properties and novel applications. Adv Mater 5:608-619

Rees GD, Robinson BH, Stefenson GR (1995) Preparativescale kinetic resolutions catalysed by microbial lipases immobilised in AOT-stabilised microemulsion-based organogels: cryoenzymology as tool for improving enantioselectivity. Biochim Biophys Acta 1259:73-81

Stamatis H, Xenakis A (1999) Biocatalysis using microemulsion-based polymer gels containing lipase. J Mol Catal B Enzym 6:399-406

Stamatis H, Xenakis A, Kolisis FN (1999) Bioorganic reactions in microemulsions: the case of lipase. Biotechnol Adv 17:293-318

Zoumpanioti M, Karali M, Xenakis A, Stamatis H (2006) Lipase biocatalytic processes in surfactant free microemulsion-like ternary systems and related organogels. Enzyme Microb Technol 39:531-539 